\title{
Infective Endocarditis Presenting as Complete Heart Block With an Unexpected Finding of a Cardiac Abscess and Purulent Pericarditis
}

\author{
Randolph E. Brown ${ }^{\mathrm{a}, \mathrm{c}}$, John Michael Chua Chiaco ${ }^{\mathrm{a}}$, Jessica L. Dillon ${ }^{\mathrm{b}}$, Edward Catherwood ${ }^{\mathrm{a}}$, \\ Kim Ornvold ${ }^{b}$
}

\begin{abstract}
Intracardiac abscess resulting in complete heart block is an infrequent complication of infective endocarditis. Most presentations of endocarditis are limited to valvular and perivalvular structures, with varying degrees of heart block occurring in the minority of cases. We report a case of endocarditis manifesting as chest pain associated with ST segment elevation and complete heart block. The patient expired unexpectedly within a few hours of presentation. Postmortem examination revealed an atrial septal abscess, purulent pericardial collection, and fibrinous pericarditis. Spread of the abscess into the atrial septum was postulated to be the cause of the complete heart block. In endocarditis, the ominous development of heart block and a poor response to antibiotic therapy imply significant extension of the infection. Management therefore requires prompt ventricular pacing with consideration for valve replacement and possible pericardial drainage.
\end{abstract}

Keywords: Infective endocarditis; Myocardial abscess; Heart block; Purulent pericarditis

\section{Introduction}

Myocardial abscess is a suppurative complication of infective endocarditis (IE) with potentially fatal outcomes. The precise incidence is unknown due to variations in reporting and best estimates can only be derived from IE figures. About 10,000 new cases of IE are diagnosed in the US each year, and of these, $30-40 \%$ are perivalvular abscesses. The aortic valve is the most commonly involved structure, followed by the mitral valve. The frequency of interatrial septal abscess is not known;

Manuscript accepted for publication July 03, 2015

aDepartment of Cardiology, Dartmouth-Hitchcock Medical Center, One Medical Center Drive, Lebanon, NH 03756, USA

bepartment of Pathology, Dartmouth-Hitchcock Medical Center, One Medical Center Drive, Lebanon, NH 03756, USA

${ }^{\mathrm{c} C}$ Corresponding Author: Randolph Eamonn Brown, Department of Cardiology, Dartmouth-Hitchcock Medical Center, One Medical Center Drive, Lebanon, NH 03756, USA. Email: randolph.e.brown@hitchcock.org

doi: http://dx.doi.org/10.14740/jocmr2228w however, five previous cases have been described after a review of the literature.

\section{Case Report}

A 63-year-old Caucasian male with a history of substance abuse, metastatic liver cancer, hepatitis $\mathrm{C}$, diabetes and peripheral arterial disease, presented to our emergency room with a 5 -day history of atypical chest pain and dyspnea.

The severe chest pain was associated with light headedness and worsened with inspiration. There was mild dyspnea, but no change in his baseline abdominal swelling and pedal edema. He denied fever, cough and recent foreign travel.

Previously, this chronic smoker abused intravenous (IV) and intranasal illicit drugs. He was surgically treated for diabetic foot ulcers on several occasions and ultimately required a right below knee amputation. Two years prior to presentation, imaging for abdominal pain revealed metastatic hepatocellular cancer.

Upon presentation, the patient was jaundiced and cachectic with tense abdominal ascites and peripheral edema. He was hypotensive with a blood pressure of $81 / 45 \mathrm{~mm} \mathrm{Hg}$, bradycardic with a pulse of $41 \mathrm{bpm}$, afebrile, and maintaining adequate oxygen saturation on room air. His cardiopulmonary examination was unrevealing.

Labs were remarkable for leukocytosis, hyponatremia, mild acidosis and deranged liver function tests (Table 1).

The patient's electrocardiogram showed complete heart block with a junctional escape rhythm, an old septal infarct and diffuse ST elevations suggestive of pericarditis (Fig. 1). A limited echocardiogram revealed preserved left ventricular systolic function, no significant valvular disease and an unremarkable pericardium. Coronary angiography showed $75 \%$ left anterior descending stenosis and 50\% right coronary artery stenosis. Because of the patient's multiple comorbidities and suspicion that his symptoms were of a non-ischemic etiology, intervention was deferred.

The patient was transferred to the coronary care unit where a transvenous pacemaker was placed for the heart block. Blood cultures were drawn and a diagnostic paracentesis was performed for the possibility of sepsis.

The hypotension of $78 / 40 \mathrm{~mm} \mathrm{Hg}$ was attributed to a combination of cirrhosis in the setting of possible sepsis. He was 
Table 1. Pertinent Laboratory Data

\begin{tabular}{|c|c|c|}
\hline Variable & Labs on admission & Reference range \\
\hline \multicolumn{3}{|l|}{ Blood count } \\
\hline Hemoglobin (g/dL) & 12.8 & $13.7-17.5$ \\
\hline White blood cell count $\left(\times 10^{3} / \mu \mathrm{L}\right)$ & 17.3 & $4-10$ \\
\hline INR & 1.3 & $0.9-1.1$ \\
\hline \multicolumn{3}{|l|}{ Chemistry } \\
\hline Sodium $(\mathrm{mmol} / \mathrm{L})$ & 123 & $135-145$ \\
\hline Potassium (mmol/L) & 5.1 & $3.5-5$ \\
\hline $\mathrm{HCO}_{3}(\mathrm{mmol} / \mathrm{L})$ & 19 & $22-31$ \\
\hline BUN (mg/dL) & 32 & $10-20$ \\
\hline Creatinine $(\mathrm{mg} / \mathrm{dL})$ & 1.36 & $0.8-1.5$ \\
\hline Glucose (mg/dL) & 154 & $65-99$ \\
\hline Lactate $(\mathrm{mmol} / \mathrm{L})$ & 10.3 & $0.5-2.2$ \\
\hline \multicolumn{3}{|l|}{ Liver function tests } \\
\hline Total protein $(\mathrm{g} / \mathrm{dL})$ & 2.3 & $0.2-1.3$ \\
\hline Albumin $(g / d L)$ & 1.9 & $3.2-5.2$ \\
\hline Total Bili (mg/dL) & 2.3 & $0.2-1.3$ \\
\hline Alk Phos (unit/L) & 199 & $40-120$ \\
\hline AST (unit/L) & 67 & $0-39$ \\
\hline ALT (unit/L) & 71 & $0-55$ \\
\hline \multicolumn{3}{|l|}{ Cardiac markers } \\
\hline Troponin T (ng/mL) & 0.1 & $<0.03$ \\
\hline Creatine kinase (unit/L) & 89 & $0-200$ \\
\hline
\end{tabular}

INR: international normalized ratio; $\mathrm{HCO}_{3}$ : bicarbonate; BUN: blood urea nitrogen; AST: aspartate aminotransferase; ALT: alanine aminotransferase.

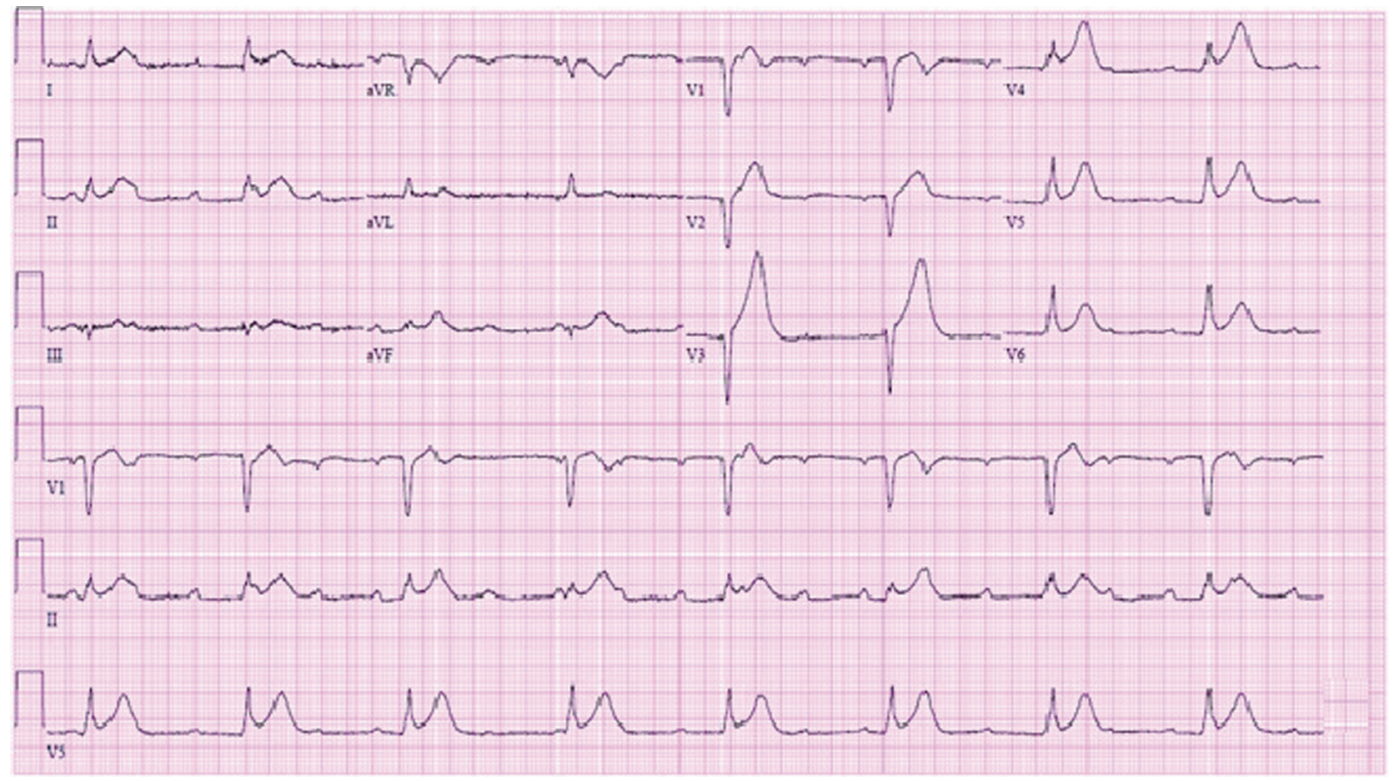

Figure 1. EKG in emergency room showing complete heart block with junctional escape at $48 \mathrm{bpm}, \mathrm{Q}$ waves in V1-V2 and diffuse ST segment elevations. 


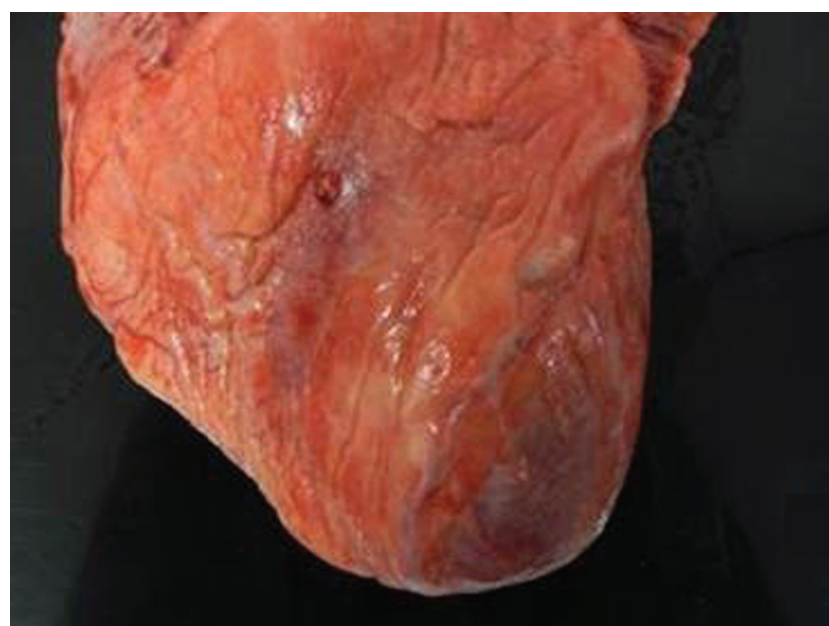

Figure 2. Gray exudate on granular epicardial surface consistent with fibrinopurulent pericarditis.

managed supportively with analgesics and IV fluid boluses. However, despite these efforts, the patient became unresponsive with pulseless electrical activity (PEA).

Cardiopulmonary resuscitation was immediately initiated with chest compressions and multiple doses of epinephrine. The ventricular fibrillation (VF) which soon followed, failed to respond to attempts at defibrillation. Ultimately, the patient eventually expired after 35 min of resuscitation.

Postmortem cultures of his ascitic fluid returned negative, but blood cultures were remarkable for methicillin-sensitive Staphylococcus aureus (MSSA).

Postmortem examination revealed $85 \mathrm{~mL}$ of brown fluid in the pericardial sac. The visceral pericardium was roughened, granular, and microscopically demonstrated a fibrinopurulent exudate (Fig. 2). The pericardial sac contained neutrophils and clusters of coliform bacteria, consistent with purulent pericarditis (Fig. 3). The mitral valves were heavily calcified, but

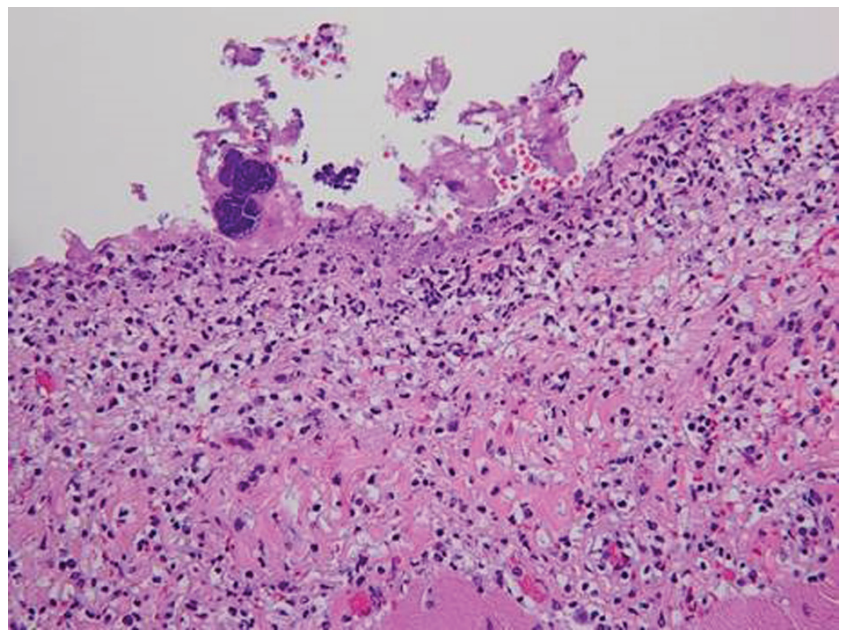

Figure 3. Photomicrograph (original magnification $\times 250$ ) of the pericardium with severe inflammation, neutrophilic infiltration and fibrin with entrapped clusters of bacteria.

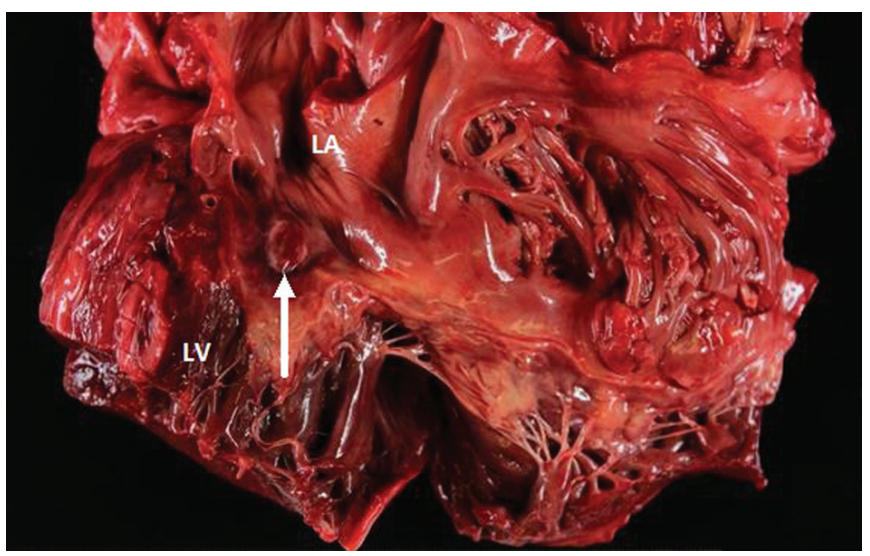

Figure 4. Gross view of the heart showing the abscess in the atrial septal wall (arrow). LV: left ventricle. LA: left atrium.

without vegetations. The atrial septum contained a $1.5 \times 1.3 \times$ $0.8 \mathrm{~cm}$ intramyocardial soft mass that spanned the full thickness of the septal wall (Fig. 4). On histologic examination, the myocardial mass displayed clusters of coliform bacteria, neutrophils, and dystrophic calcification, consistent with an intramyocardial abscess (Fig. 4). Additional pertinent autopsy findings include metastatic hepatocellular carcinoma to the adrenal glands bilaterally, portal vein thrombosis with tumor, and significant pulmonary congestion.

\section{Discussion}

Historically, IE was seen in young adults as a complication of rheumatic heart disease or poor dental hygiene. Today, with an aging population, the higher prevalence of degenerative valvular disease, multiple comorbidities, drug abuse and the use of intracardiac devices, all contribute to the current epidemic of endocarditis [1].

The incidence of IE is about seven cases per 100,000 patient years [2], and these typically involve a perivalvular abscess of the aortic valve (41\%) more so than the mitral valve (6\%) [3]. In general, cardiac abscesses are seen in 30\% of cases of native valve endocarditis and greater than $60 \%$ of cases of prosthetic valve endocarditis. However, the exact incidence of atrial septal abscesses is unknown. Our literature review has yielded five prior reports of perivalvular extension resulting in septal abscesses [4-6].

Complications of purulent pericarditis were seen in $22 \%$ of cases in one series [7] and complete heart block occurred in $11-14 \%$ of endocarditis patients [8]. Even though there have been several reports of endocarditis with a septal abscess and heart block, we found only two cases with an associated purulent pericarditis. A review of the literature has been summarized in Table 2 [4-6, 8-15].

Review of prior case reports reveal that a number of comorbidities predispose to cardiac abscesses with heart block. Underlying conditions such as congenital heart disease, valve prosthesis, immunosuppression and endocardial defects due to turbulent blood flow all set the stage for endocarditis [16]. 


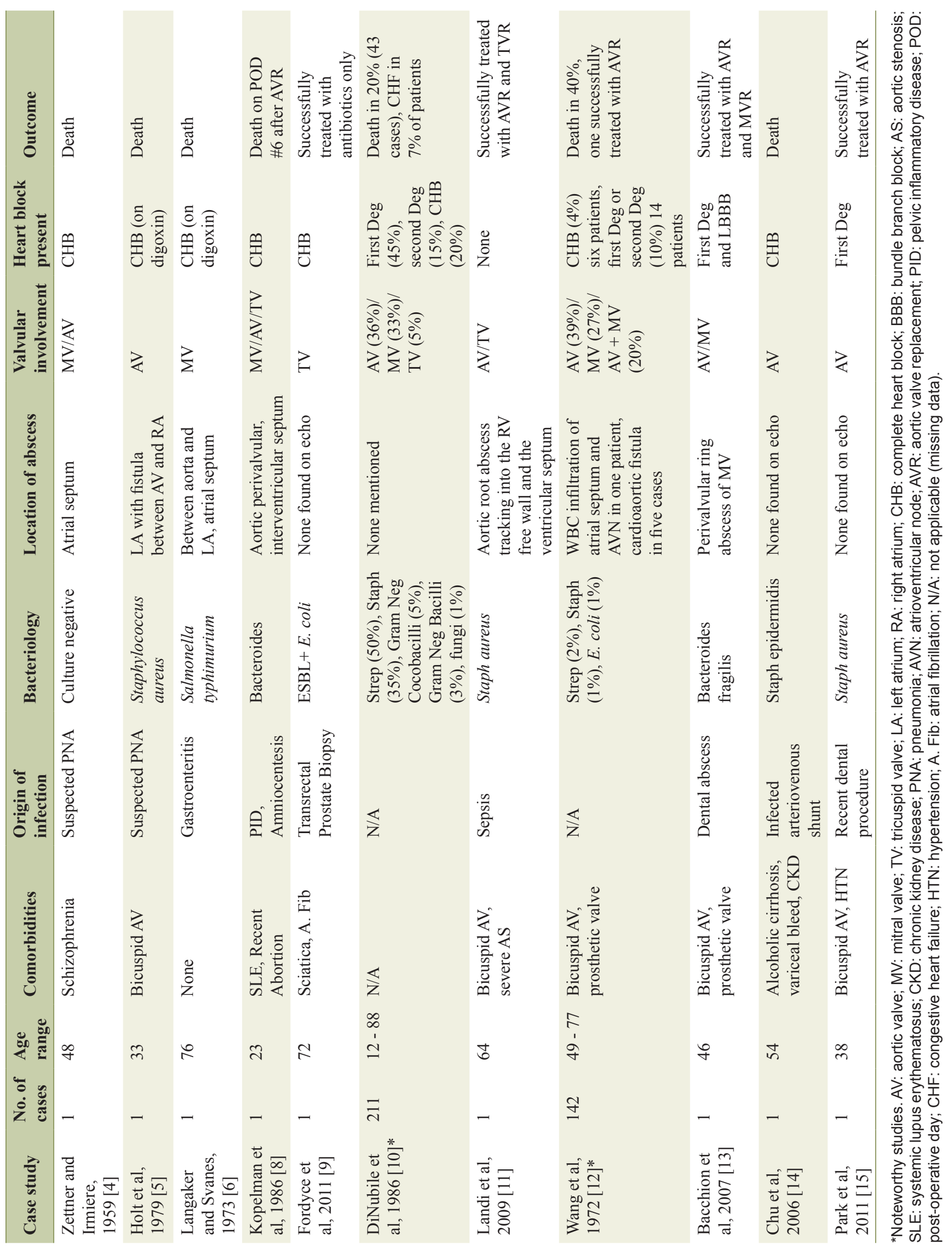


It is postulated that cardiac abscesses arise from the lodgment of infected emboli in coronary arteries $[5,6]$ or from contiguous spread from infected valves. The fact that the aortic and mitral valves share a common ring in part of their circumference and their proximity to the atrial septum explains the pattern of spread from a valvular abscess. According to Holt et al, myocardial abscesses are usually small and widespread throughout the myocardium. They are often underappreciated in IE and rarely occur in the septum.

The conduction system is contained within the right atrium and membranous septum. The left bundle branch runs along the base of the membranous septum or along the left side of the interventricular septum. Additionally, the mitral valve is located near the atrioventricular node. This may explain why abscesses of the posterior aortic sinus account for most cases of complete AV block in endocarditis [8]. Heart block is associated with $36 \%$ of aortic and $33 \%$ of mitral valve diseases [9]. There are no meta-analyses of IE with heart block. However, in a review of 142 cases of endocarditis by Wang et al, they discovered six cases (4\%) of complete heart block and 14 cases $(10 \%)$ of first or second degree heart block were reported. DiNubile et al, on the other hand, studied 211 cases of endocarditis and found that $20 \%$ had a complete heart block [10].

In the pre-antibiotic era, purulent pericarditis was due primarily to various infections such as pneumonia and endocarditis. Currently, the most common predisposing factors for hematogenous and direct contiguous spread include immunosuppression, cardiac surgery, trauma, renal disease on dialysis and substance abuse [17]. We were able to uncover two additional cases of purulent pericarditis and endocarditis. The first occurred in a 76-year-old patient with salmonella gastroenteritis who perished [6] and the second in an 18-month-old boy [18] who was successfully treated.

\section{Conclusion}

Our case is relatively unique in that our patient presented with an atrial septal abscess, complete heart block and a purulent pericarditis. The culture-positive Staphylococcus aureus in our patient is probably related to his history of IV drug use or secondary seeding from his infected amputated right leg, as streptococcus is usually more commonly found in native valve endocarditis.

This case was unusual in that, compared to other reports, there were no physical exam findings suggestive of endocarditis. The mild leukocytosis and absence of fever could be explained by his uncontrolled diabetes and metastatic cancer. However, the diffuse concave upward ST elevations on presentation were consistent with pericarditis.

In general, myocardial abscess should be suspected if there is poor response to antibiotic therapy. The development of heart block during the course of endocarditis indicates extension of the infection and implies a poor prognosis [5]. Management should therefore consist of appropriate antibiotics, ventricular pacing, surgical valve replacement and consideration for prompt pericardial drainage.

\section{Grant Support}

None.

\section{References}

1. Fuster V, et al. Hurst's The Heart. New York, NY: McGraw-Hill Companies, 2011.

2. Bonow R, et al. Braunwald's Heart Disease: A Textbook of Cardiovascular Medicine. Philadelphia, PA: Elsevier Saunders, 2012.

3. Spelman D, et al. Complications and Outcome of Infective Endocarditis. Retrieved from http://www.uptodate. $\mathrm{com} /$ contents/complications-and-outcome-of-infectiveendocarditis. 2014.

4. Zettner A, Irmiere VJ. Abscess of the heart: a complication of acute vegetative and ulcerative endocarditis. Circulation. 1959;20:590-594.

5. Holt S, Martinez AA, Coulshed N. Interatrial abscess. Postgrad Med J. 1979;55(641):207-209.

6. Langaker OM, Svanes K. Myocardial abscess due to Salmonella typhimurium. Br Heart J. 1973;35(8):871-873.

7. Guerrero M, Alvarez B, Manzarbietia F, Renedo G. Infective Endocarditis at Autopsy. Medicine Journal. 2012;3(91):152-164

8. Kopelman HA, Graham BS, Forman MB. Myocardial abscess with complete heart block complicating anaerobic infective endocarditis. Br Heart J. 1986;56(1):101104.

9. Fordyce, et al. Complete heart block associated with tricuspid valve endocarditis due to extended spectrum beta lactamase producing Escherichia coli. Can J Cardiol. 2011;27(2):26.

10. DiNubile MJ, Calderwood SB, Steinhaus DM, Karchmer AW. Cardiac conduction abnormalities complicating native valve active infective endocarditis. Am J Cardiol. 1986;58(13):1213-1217.

11. Parish LM, Liu L, Woo YJ. Endocarditis with massive aortic root abscess and atrioventricular septal destruction. Interact Cardiovasc Thorac Surg. 2009;8(2):280-282.

12. Wang K, Gobel F, Gleason DF, Edwards JE. Complete heart block complicating bacterial endocarditis. Circulation. 1972;46(5):939-947.

13. Bacchion F, Cukon S, Rizzoli G, Gerosa G, Daliento L, Thiene G, Basso C. Infective endocarditis in bicuspid aortic valve: atrioventricular block as sign of perivalvular abscess. Cardiovasc Pathol. 2007;16(4):252-255.

14. Chu CS, Sheu CC, Lee KT, Lee ST, Cheng KH, Voon WC, Sheu SH, et al. Ruptured sinus of valsalva and complete atrioventricular block complicating fulminant course of infective endocarditis: a case report and literature review. Kaohsiung J Med Sci. 2006;22(8):398-403.

15. Park MY, Jeon HK, Shim BJ, Kim HN, Lee HY, Kang JH, Kim JJ, et al. Complete Atrioventricular Block due to Infective Endocarditis of Bicuspid Aortic Valve. J Cardiovasc Ultrasound. 2011;19(3):140-143.

16. Katayama T, Tsuruya Y, Ishikawa S. Complete atrioven- 
tricular block and infective endocarditis in a patient with hypertrophic obstructive cardiomyopathy. Intern Med. 2012;51(7):749-753.

17. Santos L, et al. Purulent pericarditis: a rare diagnosis.
Rev Port Cardiol. 2013;32(9):721-727

18. Bielefeld K, O'Connor MJ, Collins RT. Primary purulent pericarditis and secondary endocarditis: a case report. Cardiol Young. 2014;24(3):563-566. 